\title{
Based on the Direction of the Antenna Pattern Measurement Level Collection Site Calibration Test Stand Development
}

\author{
Liu Xiaohu \\ Sunshine Boulevard No. 2666 college of Xinyu \\ Xinyu ,jiangxi, China \\ e-mail: xylxh163@163.com
}

\begin{abstract}
Far-field antenna pattern measurement site is important to ensure accurate measurement, and there are some problems most of the text field now, so that the test pattern data comparing simulation results with the standard existence of bias, that the development of the antenna are some obstacles. Therefore, on the basis of common antenna pattern measurement method, taking into account the special nature of the site and the actual measurement conditions, design a suitable calibration test equipment and development outdoor antenna pattern in the test field of method calibration. According to this method, it is possible for the same test site for verification testing. Level data measured obtaining contrast with the standard value in a certain range, the accuracy of verify site, and corrected the actual site.
\end{abstract}

Keywords-antenna; pattern; far field; level collection; measure

\section{INTRODUCTION}

Appeared from the antenna to now, the direction map measurement has become one of the focus topics, but now with the development of modern analytical technology and design, part of the pattern measurement becomes more and more cannot lack. Antenna design and pattern measurement always complement each other, inseparable. In order to further grasp various antenna performance and characteristics of the antenna must be measured accurately. The purpose of the antenna pattern measurement is to measure or test antenna radiation characteristics Fig 1 . The antenna beam, antenna gain and antenna sidelobe characteristics of several indexes are determined by antenna pattern. Its measurement need to rely on system to realize.

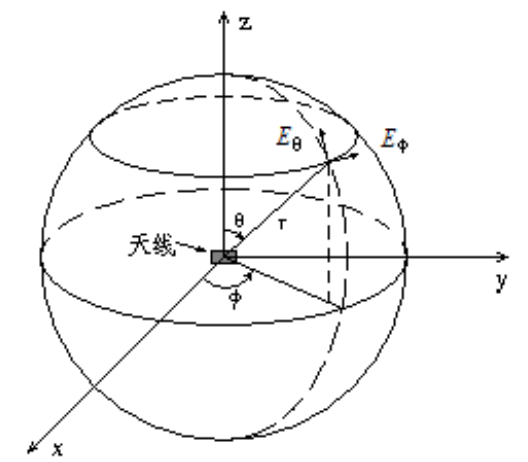

Figure 1. Coordinates of the measuring direction
Antenna test field measurement and identification parameter region of space, is an essential part of antenna testing task important condition. Because most of the antenna are working in the far field pattern, therefore, are most of the traditional antenna measurements in the far field test field. According to the applications, test the far field is usually divided into two kinds of indoor and outdoor. Microwave dark room and oblique antenna testing field were two typical representative of the test far field Fig 2. According to the principle of work, both belong to the free space test field.

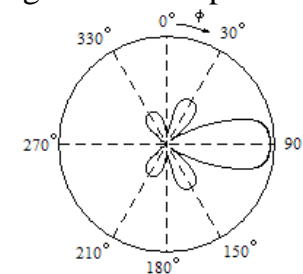

(a)

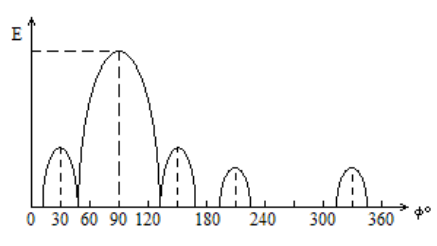

(b)
Antenna pattern test field to measure and identify the antenna parameter space region is an important condition to complete the antenna test tasks essential. Because most antenna working in the far-field antenna model, therefore, most of the conventional far-field antenna measurements in the test field. From the far-field antenna test to verify the accuracy of the site's departure, antenna test samples were analyzed by the level of floor space on the far field antenna test facility, the characteristics and laws come antenna test site to test the impact of the antenna far field, so as antenna test field correction to provide technical guidance[1].

First, the antenna pattern, the antenna radiation characteristics defined characterization (field amplitude, phase, polarization) and the spatial angular relationship of the graphic, it is an important indicator of the antenna characteristics, the direction of a complete threedimensional spatial pattern of FIG. it is a center of the sphere that the antenna phase center of the origin of coordinates, radius $r$ sufficiently large sphere[2]. Actual measurement, only the measured horizontal and vertical direction of the field amplitude to FIG. Antenna pattern can be drawn using polar coordinates, can be drawn with Cartesian coordinates. Features Polar pattern is intuitive, simple pattern can be seen from the antenna radiation field intensity spatial distribution characteristics. But the main lobe of the antenna pattern of narrow sidelobe level is low, 
the Cartesian coordinates plotted to show greater advantages[3]. Because the angle of the horizontal axis represents the vertical axis and said radiation intensity can be arbitrarily selected, even though less than 1 degree beam width can be clearly shown, and polar coordinates can not draw.

Test antenna radiation characteristics must be in the far-field region, in order to ensure sufficient precision, space-level auxiliary antenna and antenna to be tested should be kept within a certain range[2].

\section{PRODUCTION AND OPERATION OF THE TEST STAND}

The entire test frame fixed on the receiving end of the test turntable. Testing a rack fixed broadband horn antenna $(0.8-6 \mathrm{GHz})$, horn antenna can test frame rails along the three axes at a predetermined requirements of mobile (stroke are $3 \mathrm{~m}$ ), software acquisition through mobile while receiving horn antenna level data and automatically records. The entire test frame diagram below.

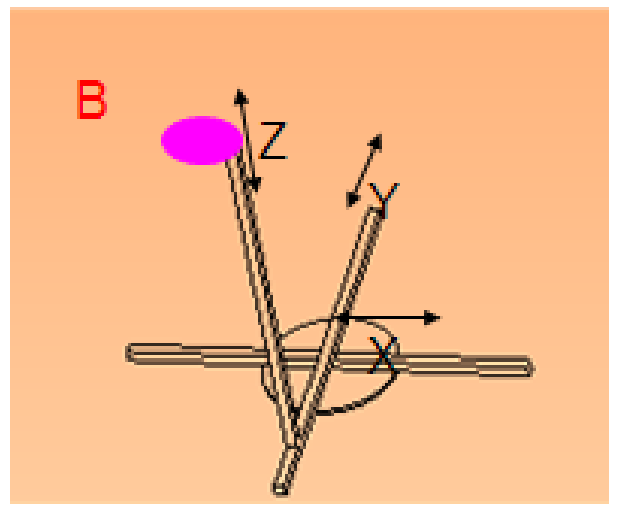

Figure 3. Schematic test stand

The main manufacturing test aircraft technical requirements below. First, the three axial size requirements stroke $3 \mathrm{~m} * 3 \mathrm{~m} * 3 \mathrm{~m}$, actual spatial dimensions not exceeding $3.5 * 3.5 * 3.75$, to cross the center of the table turntable $2 \mathrm{~m}$; motion control requirements, three axial smooth motion, no movement when the Z-axis of the horn antenna is installed after weight $1.2 \mathrm{Kg}$ to $0.5 \mathrm{~m} / \mathrm{s}$ speed reciprocating movement; Ming friction, stagnation, three axial movement driven by a motor[4], the available PC control of the three axial movement, the direction of change, speed adjustment $\mathrm{Z}$ axis support structure without significant shaking, each direction of the shift does not exceed $3 \mathrm{~cm}$.

\section{ACQUISITION SOFTWARE DEVELOPMENT AND TEST DATA}

Test software using VB, mainly because the PLC controls the motor Dodo and vector network data collection are required simultaneously. As the PC to communicate with the PLC programming with VB can be achieved between the vector and the data collection network and PC programming with $\mathrm{VB}$ is also more convenient, thus VB-testing software[5].

Here the use of Microsoft's VB 6.0 Enterprise Edition of the Chinese PC program. Visual Basic provides a procedure called an MSComm control to facilitate the design of communication serial communication. The main property of the MSComm control are: CommPort attribute is used to specify the serial port number to be used, although the Windows operating system can accommodate up to 256 serial communication ports, but VB MSComm control is limited to 16 ports; Settings property is used set initialization parameters to set a string baud rate, parity, data bits, stop bits, etc. 4 parameters, using RS-232 communication parties[6], Settings must be exactly the same, in order to successfully communicate with each other, otherwise the parties will not correctly receive the signals transmitted from each other; PortOpen attribute is used to set the communication connection status of the port. Before using the serial port serial port must first be used to open. In the PC program should be set as follows:

$$
\begin{array}{cc}
\text { Private Sub Form_Load () } & \text { 'Form } \\
\begin{array}{c}
\text { load event } \\
\text { MSComm1. CommPort }=1
\end{array} & \text { ' Choose a } \\
\text { port number } & \\
\text { MSComm1. Settings }=\text { "9600, e, 7, 2" } & \text { ' Set Port } \\
\text { PSComerties } & \text {, Open }
\end{array}
$$

Output property MSComm control provides the functionality to send, when writing data from the computer to the programmable controller or read data from the programmable controller; Input and Output Properties Properties contrast, Input property provides the ability to receive data, it the party reached the input buffer data read into the program, and clears the data buffer has been read; MSComm control to some other attribute defaults to using VB.

Are required for communication format (D8120) set during PLC computer link (proprietary protocol) and nonprotocol communication (RS instruction). Which contains the baud rate, data length, parity, stop bits, and protocol formats[7]. In modifying the D8120's settings, make sure to turn off power to the PLC, and then open it.

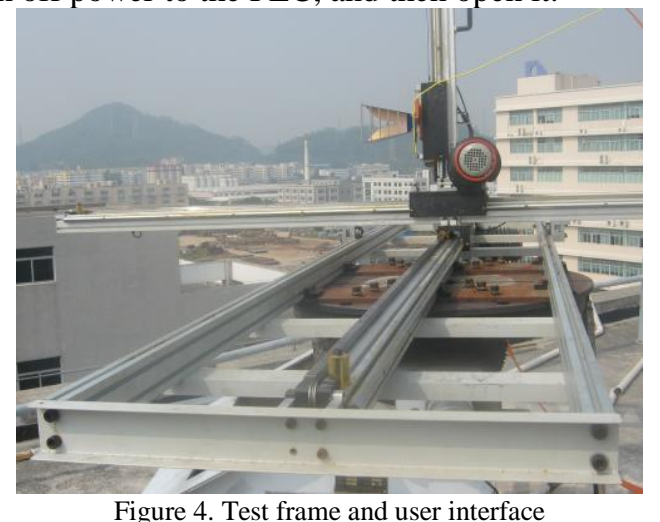

During the experiment, the speaker of the motion vector network analyzer open simultaneously for data collection, $\mathrm{X}$-axis and $\mathrm{Y}$-axis movement to the far left, followed by walking $15 \mathrm{~cm}, \mathrm{Z}$-axis is the movement from the bottom up to collect the data, the entire process of data retention in the background spreadsheet.[8] The process of data collection, to test the signal of each frequency band, polarization and time domain, a large amount of data, in the latter part of the data processing takes a lot of energy. 


\section{TEST RESULTS OF VERIFICATION AND CORRECTION VENUES}

Experiments in this paper, has been built in a far field conduct, true and reliable data collection, suitable for data analysis. During the experiment, $2 \mathrm{~F}$ and $3 \mathrm{~F}$ by collecting band, vertical and horizontal polarization, including time domain analysis of the data provided to correct the site of a strong support to ensure the correctness of the test site.

Here accordance with "GB / T 9410-2008 General technical specifications of mobile communication antenna" provides a qualified test field conditions receiving end of far-field electric field intensity should satisfy: the effective volume within the range of the antenna under test changes in the electric field strength is less than $1.5 \mathrm{~dB}$. Also check the level of the curve before and after the data, large fluctuations can occur through changes in the field antenna test the effective range of the test field can draw a clear conclusion[9].

This paper thus follows the curve plotted data, obtained through the analysis in this far-field line with fluctuations in the level of testing requirements, but there have been cases in the right level fluctuations, the actual site control found on the right side venues exist impede housing need to install the turntable absorbing material, the reflection absorption over the data. The following is a comparison of the data before and after correction, through this amendment, the site meets the environmental standards[10].

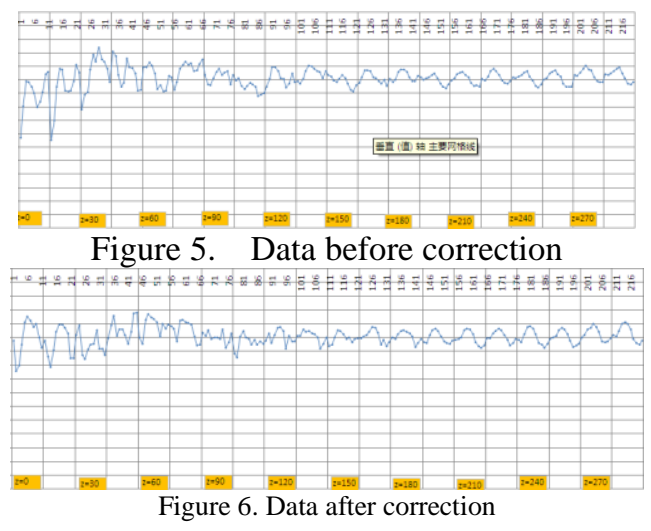

In two antenna test respectively on the double ridged horn antenna far field pattern of testing. Choose the test frequency is respectively, polarization were selected as the horizontal polarization and vertical polarization. Due to space limitations, here only part of the pattern and the related data is presented.

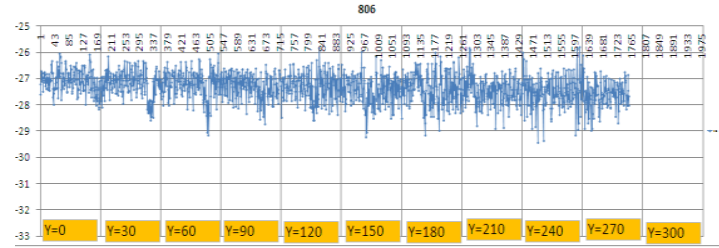

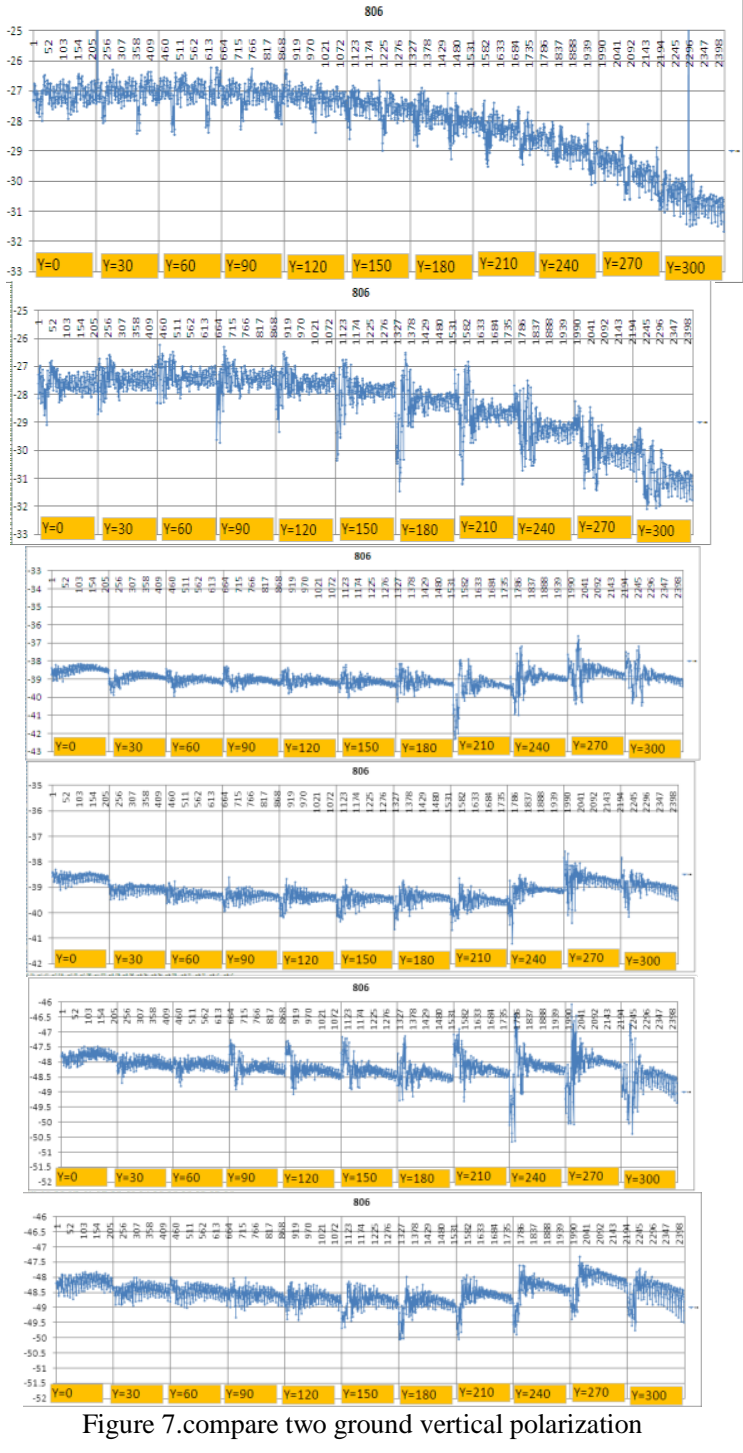

\section{CONCLUSION}

Through the test stand data acquisition, site verification to achieve a far-field antenna pattern, as the experience of other venues checksum accumulation, and has a certain reference value.

\section{REFERENCES}

[1] Rothacher, M., W. Gurtner, S. Schaer, R. Weber, W.Schlüter, H.O Hase (1995b). Azimuth- and Elevation-Dependent Phase Center Corrections for Geodetic GPS Antennas Estimated from GPS Calibration Campaigns.In: Beutler, G. et al. (Eds.). GPS Trends in Precise Terrestrial, Airborne, and Spaceborne Applications. IAG Symposium, No. 113, July 3-4, 1995, Boulder, Colorado, USA

[2] Schupler, B.R., T.A. Clark, (1991). How Different Antennas Affect the GPS Observable. GPS World, 2, No. 10 , November/December.

[3] Schupler, B.R., T.A. Clark, R.L. Allshouse (1995) Characterizations of GPS User Antennas:Reanalysis and New Results. In: Beutler, G. etal. (Eds.). GPS Trends in Precise Terrestrial,Airborne, and Spaceborne Applications. IAG Symposium, No. 113, July 3-4, 1995, Boulder, Colorado, USA..

[4] UNAVCO (1995). Receiver and Antenna Test Report. University Navstar Consortium (UNAVCO) Academic Research Infrastructure (ARI),Boulder, Colorado

[5] Wübbena, G. (1989). The GPS Adjustment Software Package GEONAP-Concepts and Models.Proceedings of the Fifth 
International Symposium on Satellite Positioning, Las Cruces, New Mexico, 452-461.

[6] Huan yuxue, "Smart antenna technology" Wireless communication technology, 2006,32(4):27-29

[7] Peng shusheng Xu zicai, Antenna pattern test instrument control and data acquisition system, Electronic technology and application 1993,(11):32-33.
[8] Li changlu, Antenna measurement technology, Chengdu telecom engineering college press 1987

[9] Dai qing Huang jijun, Modern microwave and antenna measurement technique, Beijing electronic industry publishing house 2008 .

[10] Weng jian Hou minsheng, "The design of the antenna pattern of automatic test system," Electronic engineer 2007,33(7):3. 